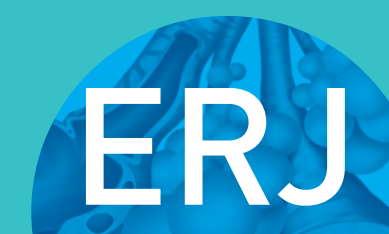

open research
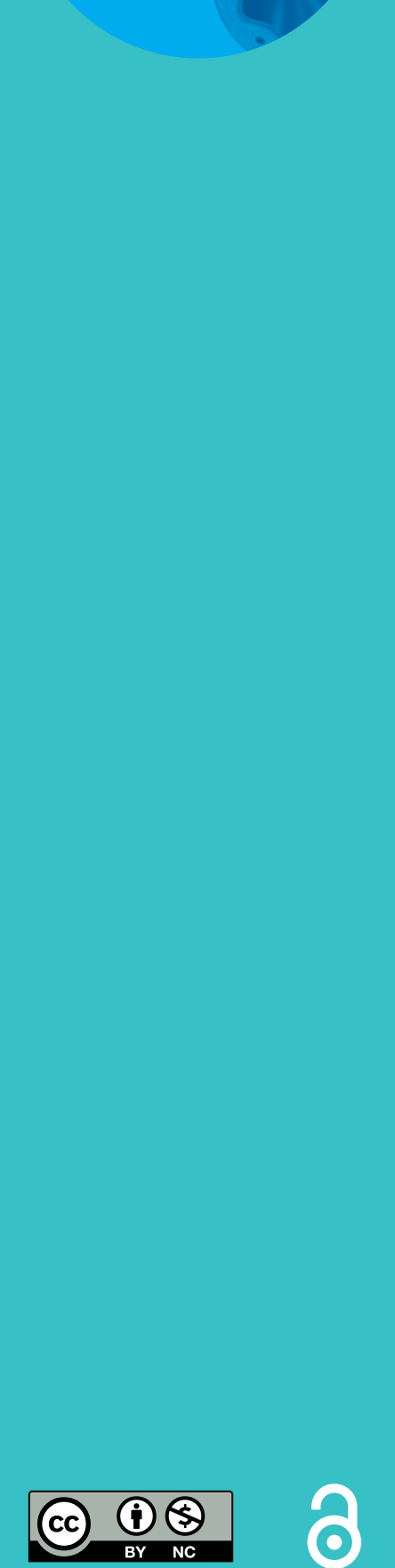

\section{Electromagnetic inductance plethysmography is well suited to measure tidal breathing in infants}

\author{
Mariann H.L. Bentsen ${ }^{1,2}$, Morten Eriksen ${ }^{3}$, Merete S. Olsen ${ }^{1}$, \\ Trond Markestad ${ }^{1,2}$ and Thomas Halvorsen ${ }^{1,2}$
}

Affiliations: ${ }^{1}$ Dept of Pediatrics, Haukeland University Hospital, Bergen, Norway. ${ }^{2}$ Dept of Clinical Science, Faculty of Medicine and Odontology, University of Bergen, Bergen, Norway. ${ }^{3}$ VoluSense AS, Bergen, Norway.

Correspondence: Mariann H.L. Bentsen, Department of Pediatrics, Haukeland University Hospital, N-5021, Bergen, Norway. E-mail: bensahelse-bergen.no

ABSTRACT Reliable, accurate and noninvasive methods for measuring lung function in infants are desirable. Electromagnetic inductance plethysmography has been used to perform infant spirometry and VoluSense Pediatrics (VSP) (VoluSense, Bergen, Norway) represents an updated version of this technique. We aimed to examine its accuracy compared to a validated system measuring airflow via a facemask using an ultrasonic flowmeter.

We tested 30 infants with postmenstrual ages between 36 to 43 weeks and weights from 2.3 to $4.8 \mathrm{~kg}$, applying both methods simultaneously and applying VSP alone. Agreement between the methods was calculated using Bland-Altman analyses and we also estimated the effect of applying the mask.

Mean differences for all breathing parameters were within $\pm 5.5 \%$ and limits of agreement between the two methods were acceptable, except perhaps for peak tidal expiratory flow (PTEF). Application of the facemask significantly increased tidal volume, minute ventilation, PTEF, the ratio of inspiratory to expiratory time and the ratio of expiratory flow at $50 \%$ of expired volume to PTEF.

VSP accurately measured tidal breathing parameters and seems well suited for tidal breathing measurements in infants under treatment with equipment that precludes the use of a facemask.

@ERSpublications

Accuracy of VoluSense Pediatrics

http://ow.ly/BIFS304sheG

This article has supplementary material available from openres.ersjournals.com

Received: May 312016 | Accepted after revision: Aug 312016

Support statement: The major funding institutions were the Western Norway Regional Health Authority and University of Bergen. The vests were sponsored by the manufacturer (VoluSense, Bergen, Norway) but the rest of the equipment used (VoluSense Pediatrics) was owned by Haukeland University Hospital. Funding information for this article has been deposited with the Open Funder Registry.

Conflict of interest: Disclosures can be found alongside this article at openres.ersjournals.com

Copyright $\odot$ ERS 2016. This article is open access and distributed under the terms of the Creative Commons Attribution Non-Commercial Licence 4.0. 


\section{Introduction}

Tidal breathing measurements and monitoring provide valuable information about lung function in infants, and can be used to evaluate lung development and management of respiratory diseases $[1,2]$. However, these measurements are currently largely used as research tools because most techniques are complex and time-consuming, require sedation, or involve the application of a facemask that adds dead space and alters the breathing pattern of the infant $[1,3-6]$.

Electromagnetic inductance plethysmography (EIP) provides a relatively novel and noninvasive supplement to previous and more intrusive methods. The EIP system consists of an electromagnet and a patient vest encircling the torso in order to quantify chest and abdominal volume changes. It does not require sedation, and allows for continuous and prolonged recording of respiratory data without using a facemask. EIP can be seen as an extension of respiratory inductance plethysmography (RIP), a validated technique that has been available for decades $[2,7,8]$. The clinical use of RIP has been limited by complex calibration procedures, in contrast to EIP, which allows for simple and patient-independent calibration. FloRight (VoluSense, Bergen, Norway) was the first prototype to use the EIP principle, with several studies demonstrating its accuracy and usefulness [9-13], although PeTrus et al. [14] reported relatively large tidal volume differences versus an ultrasonic flowmeter. VoluSense released an updated version of their method in 2015, called VoluSense Pediatrics (VSP), aiming to improve the accuracy of the volume measurements mainly by making changes to the patient vest and in the configuration of the magnetic field.

This study was performed in order to investigate the accuracy of tidal breathing parameters measured by VSP compared to a validated system measuring airflow via a facemask using an ultrasonic flowmeter, Exhalyzer D (Eco Medics, Duernten, Switzerland). We hypothesised that the differences between the two methods would be within $15 \%$ of each other, a range considered acceptable in a clinical setting. We also aimed to investigate how application of the facemask influenced the measurements.

\section{Methods}

\section{Participants}

Newborn infants without a need for assisted ventilation or oxygen supplementation and with a weight $\geqslant 2.0 \mathrm{~kg}$ were eligible. The weight limit was set because Exhalyzer D is not approved for children $<2 \mathrm{~kg}$. Only healthy children were recruited, as simultaneous measurements with two methods were expected to be somewhat cumbersome and time consuming. The participants were mostly recruited from the maternity clinic at Haukeland University Hospital, Bergen, Norway, and studied either just before discharge or when returning to the outpatient clinic for routine screenings. Six participants were recruited from the hospital's neonatal intensive care unit where they initially had been admitted for either prematurity, hypoglycaemia or suspected infection, but they were ready for discharge when measured. Informed written consent was obtained from the parents. The study was approved by the Regional Committee on Medical Research Ethics of Western Norway.

\section{Equipment and modifications compared to the old EIP model}

VSP consists of a patient vest shaped as a rectangle and made from very elastic fabric with two thin conductive elements attached to the outside in a wide zig-zag pattern (figure 1). The upper zig-zag wire covers the chest and the lower the abdomen, enabling separate analyses and detection of thoracoabdominal asynchrony. The vest is wrapped around the torso of the baby and fastened with Velcro. An electromagnet positioned above the cot at a precise height and parallel to the baby's spine generates a weak magnetic field that alternates with a frequency of $\sim 100 \mathrm{kHz}$. The magnetic field induces a voltage in the metal wires of the vest that is proportional to the instantaneous average cross-sectional area of the torso covered by the wires, and increases and decreases proportionally to the infant's breathing movements. The system is initially calibrated against a calibration coil with a known area $\left(A_{\mathrm{C}}\right)$ positioned at the side of the infant in the cot to establish the relationship between the induced voltage $\left(U_{\mathrm{C}}\right)$ and this area. During measurements, the thoracic or abdominal volumes are calculated as

$$
V=\frac{U_{\mathrm{M}} A_{\mathrm{C}} W_{\mathrm{Z}}}{U_{\mathrm{C}}}
$$

where $U_{\mathrm{M}}$ is the voltage that is measured and $W_{\mathrm{Z}}$ is the width of the zig-zag pattern of the conducting loop in the vest. According to Faraday's law of induction, the induced voltage of a conductor loop will be proportional to the area regardless of its shape, given a homogeneous magnetic field. The volume signal is converted by a computer into flow by numerical differentiation, and flow-volume loops and tidal breathing parameters are calculated [15]. All calculations and storage of raw data are performed at a rate of $100 \mathrm{~Hz}$. The volume variations of the torso are assumed to be caused by variations in the volume of air in the lungs. 


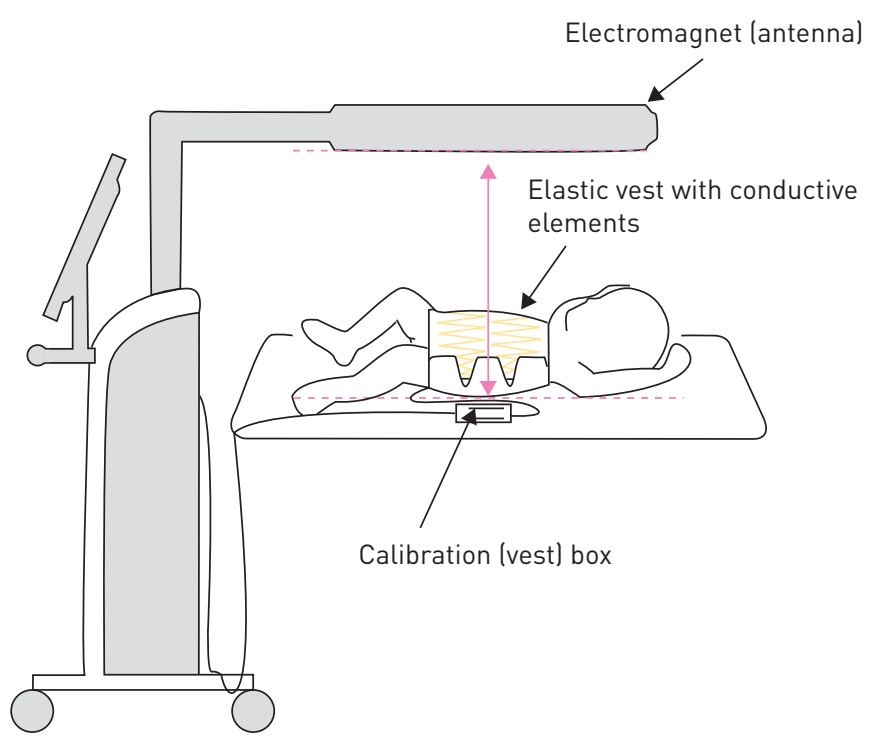

FIGURE 1 Schematic drawing of the VoluSense Pediatrics (VoluSense, Bergen, Norway) measurement system. The supine infant is wearing an elastic vest with conductive elements. An electromagnet positioned above the cot at a precise height generates a weak magnetic field that induces a voltage in the metal wires of the vest. This voltage is proportional to the cross-sectional area of the torso, and will increase and decrease proportionally to the breathing movements of the infant. The system is initially calibrated against an area reference coil positioned at the side of the infant. A computer controls the system during collection and logging of data.

Compared to FloRight, which was described in detail previously [13], VSP is different mainly in the design of the patient vest and in the configuration of the magnetic field, but these changes have also led to upgrades of hardware and software. For a detailed description of the differences between FloRight and VSP, please see the online supplementary material. An ultrasonic flowmeter (Exhalyzer D) with a flow head suitable for infants $>2 \mathrm{~kg}$ body weight and a flow-range of $\pm 0.5 \mathrm{~L} \cdot \mathrm{s}^{-1}$ was used as a reference method. The system was calibrated according to the instructions from the manufacturer with a $10-\mathrm{mL}$ volumetric syringe. A neonatal facemask with soft edges allowing a good seal was applied. The total dead space of the system (including the mask) was $9 \mathrm{~mL}$. Measurements were performed according to the latest European Respiratory Society/American Thoracic Society standards of infant lung function testing [16].

\section{Protocol/study design}

Validation of VSP was based on simultaneous measurements with VSP and Exhalyzer D, as illustrated in figure 2. The effect of the facemask was assessed by comparing the VSP measurements obtained before to those obtained after the facemask was put on. The infants were dressed in the appropriate-sized vest selected according to the length from the armpit to the hip. Care was taken to ensure that the vest fitted snugly around the torso, and that the width of the copper wire zig-zag covered the entire thorax and abdomen including the pubic region. The vest was applied directly to the skin; neither a body nor a nappy was worn underneath, but a nappy and a warm blanket were put on outside the vest. The infants were fed before being placed supine in a cot and encouraged to sleep. No sedation was used. Once the infant fell asleep, data were collected with VSP alone for 2-3 min. The VSP measurement was continued while the Exhalyzer D neonatal facemask was placed gently, but firmly, over the infant's mouth and nose, ensuring a good seal. Once the facemask was in place, a marker was inserted in the VSP recording. The Exhalyzer D measurement was started $\sim 30 \mathrm{~s}$ after the facemask was put on, to allow adaptation to the facemask. Simultaneous recording using both devices was then performed for $\sim 1 \mathrm{~min}$ before the mask was removed. New markers were inserted in the VSP recording as the Exhalyzer D measurement was started and stopped.

\section{Data analysis}

The markers in the VSP recording (figure 2) enabled visual identification of the corresponding respiratory cycles from the VSP and the Exhalyzer D recording. This allowed for comparison of the same flow, volume and timing parameters from each device. Segments with movement artefacts, apnoeas or sighs were removed from both recordings before the breathing segments were analysed using the software provided by the respective manufacturers.

Effect of the facemask was assessed by comparing the breathing parameters obtained from the VSP before versus after putting on the Exhalyzer D mask. 


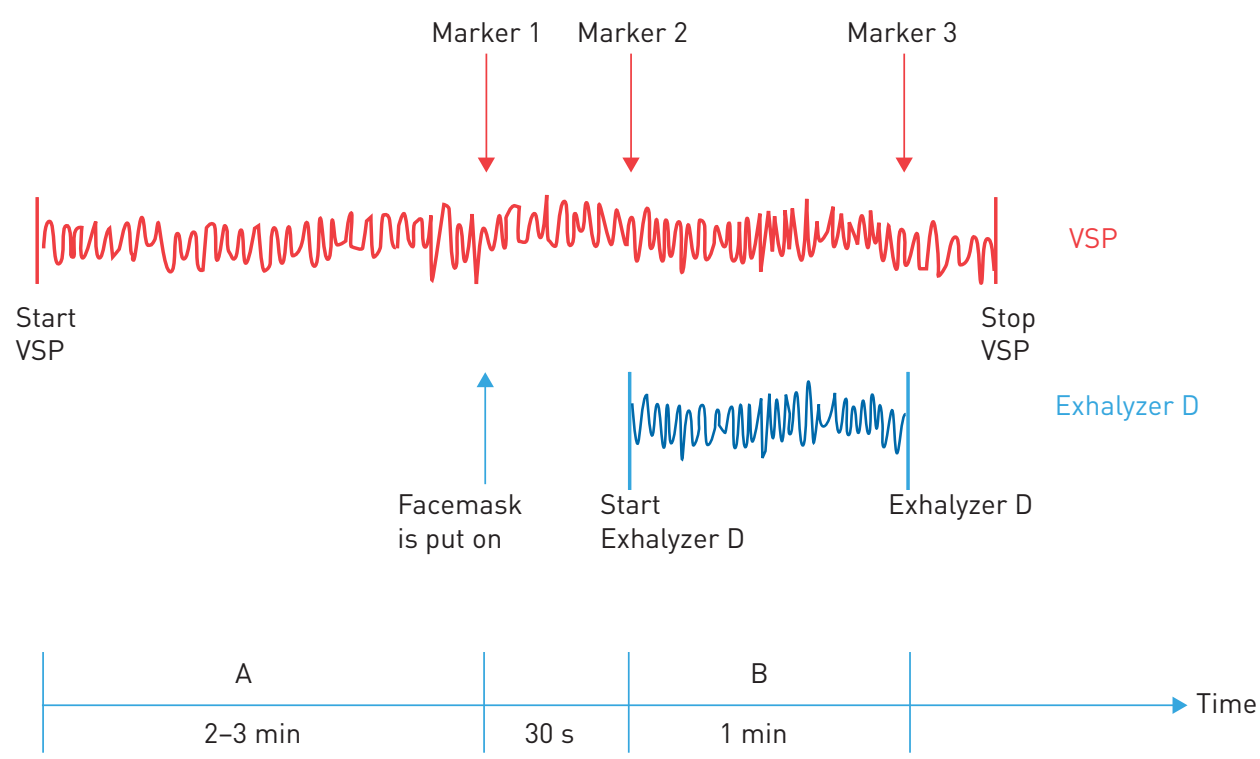

FIGURE 2 Schematic illustration of the study design. When the infant fell asleep, data were first collected with VoluSense Pediatrics (VSP) (VoluSense, Bergen, Norway) alone for 2-3 min (A) before the Exhalyzer D (Eco Medics, Duernten, Switzerland) neonatal facemask was placed over the infant's mouth and nose. Once the facemask was in place, a marker was inserted in the VSP recording. To allow adaptation to the facemask, the Exhalyzer $D$ measurement was started $\sim 30 \mathrm{~s}$ after the facemask was put on. Simultaneous recording using both devices was done for $\sim 1 \mathrm{~min}(\mathrm{~B})$ before the mask was removed. New markers were inserted in the VSP recording as the Exhalyzer D measurement was started and stopped.

\section{Statistical methods}

Data were analysed and graphs were created with SPSS version 22 (IBM SPSS Statistics, Armonk, NY, USA) and MedCalc version 13.1 (MedCalc Software, Mariakerke, Belgium).

We constructed Bland-Altman plots to visualise agreement between the two measurement techniques $[17,18]$. After the inclusion of 18 cases, the Bootstrap resampling method [17] was used to estimate the number of participants needed in the study. We concluded that the reliability of the $95 \%$ limits of agreement would not improve by including $>30$ participants in the study. One-sample t-tests were used to assess if the mean differences between data obtained with the two methods differed significantly from zero, indicating the presence of a consistent bias. To evaluate the existence of proportional bias, i.e. that the methods did not agree equally through the range of measurements, the difference between the methods was regressed on the average of the two methods. To evaluate the effect of the facemask, we compared the means of the different breathing parameters from the VSP recording with and without facemask using paired sample t-tests.

\section{Results}

\section{Participants}

30 infants with postmenstrual ages ranging between 36 and 43 weeks and a weight range of $2.3-4.8 \mathrm{~kg}$ were studied. Their characteristics are shown in table 1. Successful simultaneous measurements were obtained for all participants. In 14 cases, the simultaneous measurement was successful at the first attempt; in 13 cases, at the second attempt; and in three cases, at the third attempt. The most common cause of unsuccessful measurements was babies waking up, disapproving of application of the facemask. Other causes were movement artefacts in the VSP recordings or obvious mask leakage in the Exhalyzer D recording.

\section{TABLE 1 Characteristics of the participants $(n=30)$}

Males

Gestational age at birth weeks

Postnatal age at study days

Postmenstrual age at study weeks

Birth weight kg

Weight at study $\mathbf{k g}$

Length at study $\mathrm{cm}$
$18(60 \%)$

$38.0(36.5-39.5)$

$13.1(3.4-22.8)$

$39.9(39.2-40.6)$

$3.2(2.9-3.6)$

$3.4(3.2-3.7)$

$49.5(48.7-50.4)$

Data are presented as mean (95\% CI) unless otherwise stated. 


\section{Comparison of the two methods}

Agreements for all tidal breathing parameters are presented in figure 3.

Tidal volumes (per kg) showed a nonconsistent and nonsignificant mean difference (VSP minus Exhalyzer D) of $-0.15 \mathrm{~mL} \cdot \mathrm{kg}^{-1}$, with limits of agreement within $\pm 0.93 \mathrm{~mL} \cdot \mathrm{kg}^{-1}$. This corresponds to a difference of $-3.0 \%$ and limits of agreement within $\pm 16.0 \%$ (figure $3 \mathrm{a}$ and $\mathrm{b}$ ).

Mean minute ventilation was 3.8\% lower for VSP and the difference was consistent based on a one-sample t-test (figure 3c). Measured respiratory rates differed by $<1 \%$ (figure 3d). PTEF was higher when measured by VSP than by Exhalyzer D; on average, the difference was $+5.0 \%$. The difference was consistent but with a relatively large standard error and wide limits of agreements (figure $3 \mathrm{e}$ ).

Time to peak tidal expiratory flow $(t \mathrm{PTEF}) /$ total expiratory time ( $t \mathrm{E})$ ratio differed, on average, by $-5.4 \%$ (VSP minus Exhalyzer D), with limits of agreement of $\pm 18.0 \%$ (figure $3 \mathrm{f}$ ). Regression analysis revealed a small proportional bias of the mean difference ( $p=0.0008$, regression slope 0.51 and $r^{2}=0.33$ ). The inspiratory time $(t \mathrm{I}) / t \mathrm{E}$ ratio and the tidal expiratory flow at $50 \%$ of expired volume (TEF50)/PTEF ratio both corresponded well, with small mean differences $(-3.1 \%$ and $-3.0 \%$, respectively; VSP minus Exhalyzer D) and relatively narrow limits of agreement (figure $3 \mathrm{~g}$ and $\mathrm{h}$ ). A small proportional bias was found for both parameters ( $p=0.005$, regression slope 0.23 and $r^{2}=0.25$; and $p=0.034$, regression slope 0.38 and $r^{2}=0.15$, respectively).

\section{Influence of the facemask}

Application of the facemask significantly increased the tidal volume and the minute ventilation. $t \mathrm{PTEF}, t \mathrm{I} / \mathrm{tE}$ and TEF50/PTEF were also significantly increased. No difference was found for respiratory rate or $t \mathrm{PTEF} / \mathrm{tE}$ (table 2).

\section{Discussion}

The principle of EIP, as utilised by VSP, accurately measured tidal breathing parameters in infants when compared to data obtained from Exhalyzer D, which is based on measuring airflow through a facemask. Mean differences for all tidal breathing parameters were within $\pm 5.5 \%$. The limits of agreement between the two methods were within ranges that, in our opinion, were acceptable given a clinical context, with the possible exception of PTEF. Application of the facemask significantly increased tidal volume, minute ventilation, $t \mathrm{PTEF}, t \mathrm{I} / t \mathrm{E}$ and TEF50/PTEF.

\section{Comparison of the two methods and possible influences}

This is the first published study using VSP, and thus, comparison with previous validation data is impossible. However, compared to results from previous validation studies of FloRight, the results generated by VSP are just as accurate. Petrus et al. [14] included 37 infants in their study and found that tidal volumes, on average, were $1.3 \mathrm{~mL} \cdot \mathrm{kg}^{-1}$ lower using FloRight compared to an ultrasonic flowmeter and a facemask. This contrasts with the results of OLDEN et al. [9], who compared FloRight to an ultrasonic flowmeter, and the results of Williams et al. [11], who compared FloRight to a pneumotachograph. Both these studies found close agreement between the methods. It also contrasts with the difference of $0.15 \mathrm{~mL} \cdot \mathrm{kg}^{-1}$ that was found in the present study testing VSP. However, substantiated by these findings, and considering the fact that it is difficult to completely avoid a mask leak, which will lead to the opposite volume bias, both FloRight and VSP probably measure volumes that might be somewhat lower than the actual volumes. The most plausible reason for this is the inability of the vest to capture breathing movements at the top of the thorax, above the armpit level, as well as at the back towards the underneath surface where the magnetic field fails to create voltage changes. The accuracy of Exhalyzer D is, according to the manufacturer, better than $2 \%$ for flow- and volume-derived parameters, which is not expected to contribute substantially to the calculated mean differences between the two methods or to their limits of agreement.

For all parameters except $t \mathrm{PTEF}, t \mathrm{I} / \mathrm{tE}$ and TEF50/PTEF, we found a consistent bias, which means that the difference between the methods can be adjusted for by subtracting the mean difference from the VSP method. The proportional biases found for $t \mathrm{PTEF} / \mathrm{tE}, t \mathrm{I} / t_{\mathrm{E}}$ and TEF50/PTEF, which indicate that the limits of agreement will be wider as the numerical level of the pairwise mean increases, were small and probably of limited clinical relevance when testing infants.

Compared to the results of PETRUs et al. [14], we found smaller mean differences but somewhat wider limits of agreement for all breathing parameters. In addition, OLDEN et al. [9] found limits of agreement for tidal volume and $t \mathrm{PTEF} / \mathrm{tE}$ that were narrower than in our study. In the validation study by WiLLIAMS et al. [11], however, where FloRight was compared to a pneumotachograph, the limits of agreement were wider than in our study. In other words, studies comparing FloRight to either an ultrasonic flowmeter or a pneumotachograph show divergent results regarding the variability between paired measurements. This is as expected, as the studies have all used different "gold-standard methods". It should be emphasised that no 

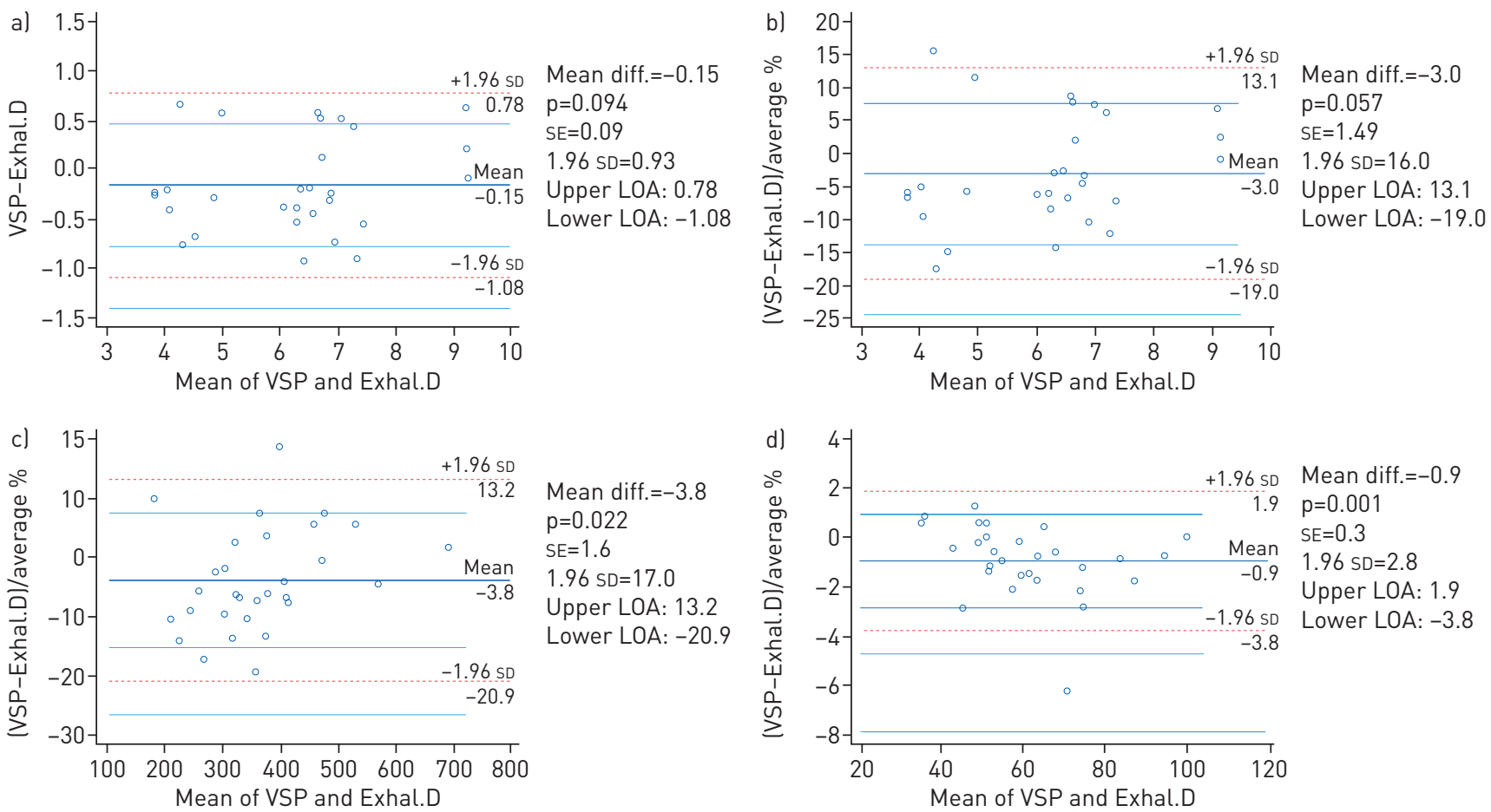

Mean diff. $=-3.8$ $\mathrm{p}=0.022$ $\mathrm{SE}=1.6$

$1.96 \mathrm{SD}=17.0$

Upper LOA: 13.2

Lower LOA: -20.9

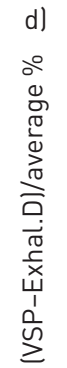
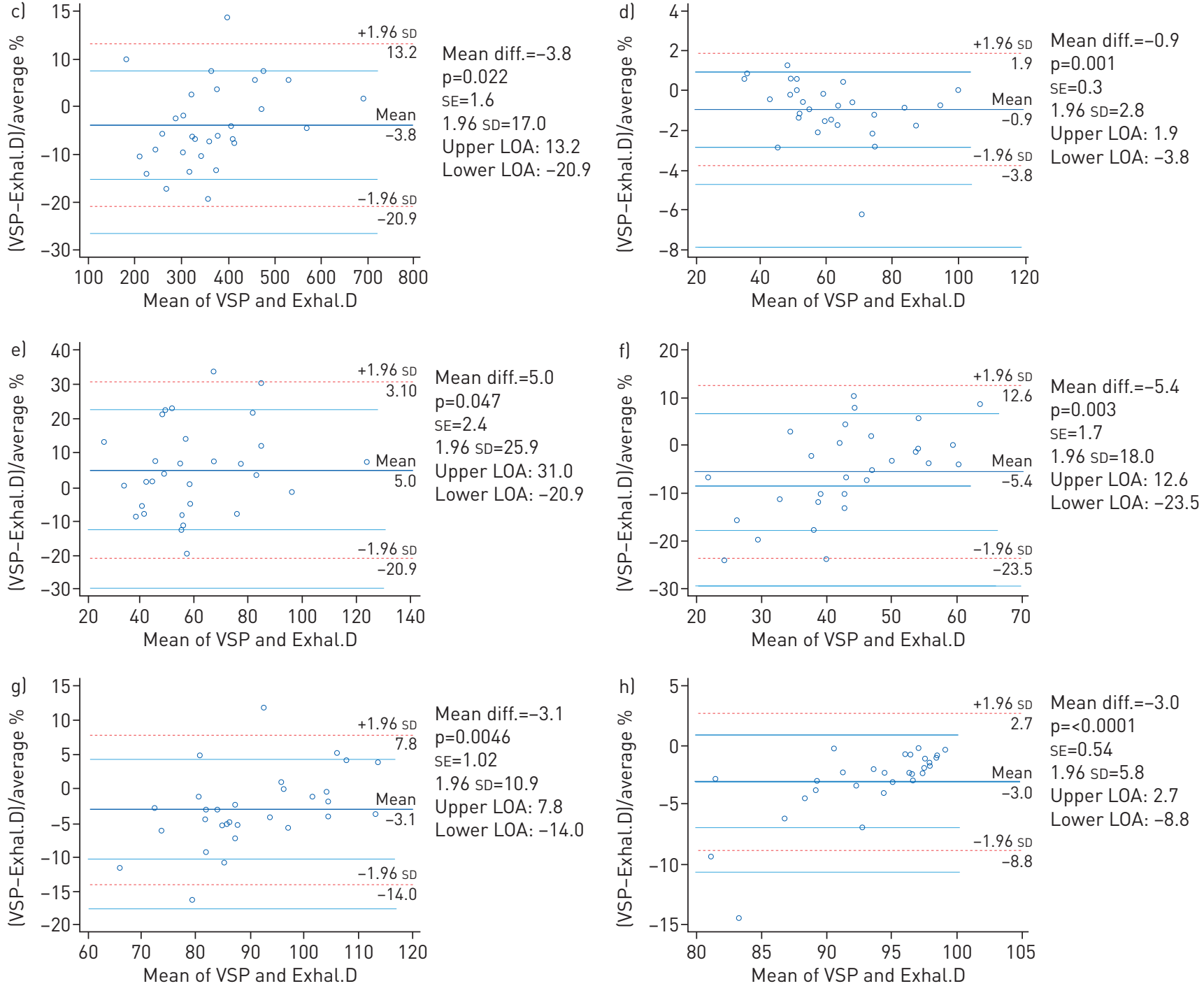

FIGURE 3 Agreement regarding tidal breathing parameters between VoluSense Pediatrics (VSP) (VoluSense, Bergen, Norway) and Exhalyzer D (Exhal.D) (Eco Medics, Duernten, Switzerland) expressed as Bland-Altman plots. a, b) Tidal volume per kg body weight; c) minute ventilation per kg body weight; d) respiratory rate (per min); e) peak tidal expiratory flow (PTEF) (mL. $\mathrm{s}^{-1}$ ); f) time to PTEF/expiratory time (tE) ratio (\%); g) inspiratory time/tE (\%); h) tidal expiratory flow at $50 \%$ of expiratory volume/PTEF ratio (\%). Horizontal axes represent the mean of the two measurement values ((VSP+Exhal.D)/2). Vertical axes: a) difference in tidal volume between the two methods as raw data (VSP minus Exhal.D); $b-h$ ) differences as percentages of their mean value, i.e. the relative difference between the methods. $\mathrm{p}$-values are from one-sample t-tests, testing the mean difference between the two methods against zero. Mean diff.: mean difference between the two methods; LOA: limits of agreement.

gold-standard method can be expected to produce a "true value" for infant airflow variables. Thus, the limits of agreement reflect how much the two methods differ from each other for most individuals, not how much VSP differs from the true value. 


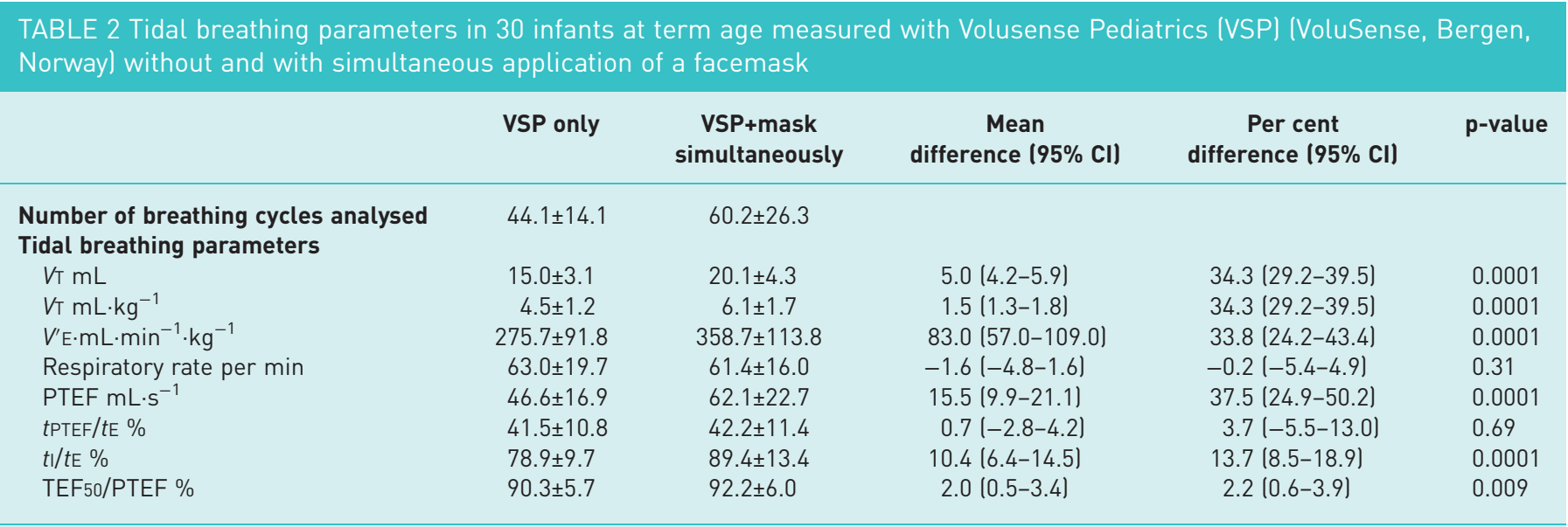

Data are presented as mean \pm SD unless otherwise stated. VT: tidal volume; $V$ 'E: minute ventilation. PTEF: peak tidal expiratory flow; tPTEF: time to peak tidal expiratory flow; $t E$ : total expiratory time; $t I$ : inspiratory time; TEF50: tidal expiratory flow at $50 \%$ of expiratory volume. $p$-values are from paired-sample t-tests.

For PTEF, the limits of agreement were relatively wide in the present study, indicating that the differences between the two methods varied relatively much among the participants. One possible explanation may have been variable degrees of air leakage through the Exhalyzer D facemask, which is most likely to occur during peak expiration and therefore particularly likely to influence PTEF. The VSP recordings may have been influenced by unnoticed and variable use of accessory respiratory muscles being incorrectly recognised as thoracic volume changes, although most such artefacts are easily recognised. Moreover, the VSP measurements could have been influenced by variable displacement of venous truncal blood volume into the head and limbs during respiration. VSP calculates flow from volume changes based on the assumption that all internal volume variation of the torso is caused by airflow within the respiratory system, and that all such airflow induces corresponding external volume variations of the torso. These are small but not insignificant simplifications that can explain some of the discrepancies we found for most of the tidal breathing parameters. Precise control over these issues requires whole-body plethysmography.

Software differences are also a potential source of bias that may influence the agreement between the two measurement methods. Determining the beginning and end of inspiration and expiration is a well-known problem when it comes to computer-based analysis of tidal breathing [18]. We believe that Exhalyzer D interprets the start of expiration slightly later than VSP. Such phase shifts can explain some of the minor differences found for $t \mathrm{PTEF} / t \mathrm{E}, \mathrm{tI} / \mathrm{tE}$ and TEF50/PTEF.

\section{Strengths and limitations of VSP}

The major weakness of the EIP method is the high sensitivity to a poor fit of the vest. It must be applied directly to the skin, as the fabric has to enclose the skin tightly with no wrinkles. Any body surface moving with respiration that is not sufficiently covered by the vest will lead to an underestimation of measured volumes. As a consequence, the infants must have their hips extended during the measurements, because flexion of the hips will cause folding of the fabric. The experience of the personnel performing the measurements will therefore have an impact on the accuracy of the results [13]. Skin lesions like surgical wounds, gastroschisis or stomas and drainage tubes will limit its clinical application.

The major advantage of the method is the avoidance of a facemask. This allows extended recordings in babies who are respiratory unstable or use equipment that may preclude the use of a facemask, primarily continuous positive airway pressure (CPAP) or high-flow nasal cannulas (HFNCs). In accordance with previous studies, we found that applying a facemask significantly increased minute ventilation [2, 4, 7]. This finding is physiologically plausible and most likely explained by increased dead space ventilation induced by the facemask or by tactile stimulation of the facial skin. We found that parameters reflecting airway obstruction, i.e. $t \mathrm{t} / \mathrm{tE}$ and TEF50/PTEF, were also significantly increased by the facemask. We believe that this apparent "anti-obstructive effect" was caused by a slight positive expiratory counter pressure generated mainly by the resistance of the flowmeter. This might increase the average diameter of small and medium-sized airways, and thereby cause tE to decrease and TEF50 to increase, which is in accordance with our findings. 


\section{Conclusions}

VSP accurately measures tidal breathing parameters if used correctly by experienced personnel. As inductive plethysmography does not involve a facemask, it can be applied in infants needing respiratory support such as CPAP and HFNCs. Thus, VSP is likely to be well suited for neonatal intensive care units and hospital wards treating children with pulmonary illnesses such as bronchiolitis. However, validation studies and normative data from children of all weight classes, and by gestational age, weight and sex will be needed. The validity of the relatively few existing sets of reference equations for infant spirometry parameters is probably limited to the methods that were used to obtain them [19-21].

\section{Acknowledgements}

We would like to thank the infants who participated in this study and their parents.

\section{References}

1 Baldwin DN, Pillow JJ, Stocks J, et al. Lung-function tests in neonates and infants with chronic lung disease: tidal breathing and respiratory control. Pediatr Pulmonol 2006; 41: 391-419.

2 Reiterer F, Sivieri E, Abbasi S. Evaluation of bedside pulmonary function in the neonate: from the past to the future. Pediatr Pulmonol 2015; 50: 1039-1050.

3 Schmalisch G, Foitzik B, Wauer RR, et al. Effect of apparatus dead space on breathing parameters in newborns: "flow-through" versus conventional techniques. Eur Respir J 2001; 17: 108-114.

4 Fleming PJ, Levine MR, Goncalves A. Changes in respiratory pattern resulting from the use of a facemask to record respiration in newborn infants. Pediatr Res 1982; 16: 1031-1034.

5 Dolfin T, Duffty P, Wilkes D, et al. Effects of a face mask and pneumotachograph on breathing in sleeping infants. Am Rev Respir Dis 1983; 128: 977-979.

6 Marsh MJ, Ingram D, Milner AD. The effect of instrumental dead space on measurement of breathing pattern and pulmonary mechanics in the newborn. Pediatr Pulmonol 1993; 16: 316-322.

7 Ali N, Claure N, Alegria X, et al. Effects of non-invasive pressure support ventilation (NI-PSV) on ventilation and respiratory effort in very low birth weight infants. Pediatr Pulmonol 2007; 42: 704-710.

8 Lavizzari A, Veneroni C, Colnaghi M, et al. Respiratory mechanics during NCPAP and HHHFNC at equa distending pressures. Arch Dis Child Fetal Neonatal Ed 2014; 99: F315-F320.

9 Olden C, Symes E, Seddon P. Measuring tidal breathing parameters using a volumetric vest in neonates with and without lung disease. Pediatr Pulmonol 2010; 45: 1070-1075.

10 Pickerd N, Williams EM, Kotecha S. Electromagnetic inductance plethysmography to measure tidal breathing in preterm and term infants. Pediatr Pulmonol 2013; 48: 160-167.

11 Williams EM, Pickerd N, Eriksen M, et al. Estimation of tidal ventilation in preterm and term newborn infants using electromagnetic inductance plethysmography. Physiol Meas 2011; 32: 1833-1845.

12 Pickerd N, Williams EM, Watkins WJ, et al. Tidal breathing in preterm infants receiving and weaning from continuous positive airway pressure. J Pediatr 2014; 164: 1058-1063.

13 Bentsen $\mathrm{MH}$, Haaland OA, Lonning LB, et al. A new non-invasive method of infant spirometry demonstrates a level of repeatability that is comparable to traditional methods. Acta Paediatr 2015; 104: 1130-1137.

14 Petrus NC, Thamrin C, Fuchs O, et al. Accuracy of tidal breathing measurement of floright compared to an ultrasonic flowmeter in infants. Pediatr Pulmonol 2014; 4: 380-388.

15 Eriksen M. Coil system and method for obtaining volumetric physiological measurements. US patent number US 8328739B2 2012.

16 Frey U, Stocks J, Coates A, et al. Specifications for equipment used for infant pulmonary function testing. ERS/ ATS Task Force on Standards for Infant Respiratory Function Testing. European Respiratory Society/American Thoracic Society. Eur Respir J 2000; 16: 731-740

17 Pezzullo J. Biostatistics for Dummies. Hoboken, John Wiley \& Sons, 2013.

18 Bates JH, Schmalisch G, Filbrun D, et al. Tidal breath analysis for infant pulmonary function testing. ERS/ATS Task Force on Standards for Infant Respiratory Function Testing. European Respiratory Society/American Thoracic Society. Eur Respir J 2000; 16: 1180-1192.

19 Fuchs O, Latzin P, Thamrin C, et al. Normative data for lung function and exhaled nitric oxide in unsedated healthy infants. Eur Respir J 2011; 37: 1208-1216.

20 Hulskamp G, Lum S, Stocks J, et al. Association of prematurity, lung disease and body size with lung volume and ventilation inhomogeneity in unsedated neonates: a multicentre study. Thorax 2009; 64: 240-245.

21 Latzin P, Roth S, Thamrin C, et al. Lung volume, breathing pattern and ventilation inhomogeneity in preterm and term infants. PLoS One 2009; 4: e4635. 